\section{A New Potential Contrast Agent for Magnetic Resonance Imaging: Iron Oxide-4A Nanocomposite}

\author{
Zareei L. ${ }^{1,2}$, Divband B. ${ }^{3}$, Mesbahi A. ${ }^{4}$, Khatamian M. ${ }^{5}$, Kiani \\ A. ${ }^{6}$, Gharehaghaji N. ${ }^{7 *}$
}

\begin{abstract}
Background: Magnetic resonance imaging (MRI) contrast agents have an important role to differentiate healthy and diseased tissues. Access and design new contrast agents for the optimal use of MRI are necessary. This study aims to evaluate iron oxide-4A nanocomposite ability to act as a magnetic resonance imaging contrast agent.
\end{abstract}

Material and Methods: Iron oxide-4A nanocomposite (F4A) was synthesized. MTT assay was used to consider the nanocomposite safety for cell culture. The $\mathrm{T} 1$ and $\mathrm{T} 2$ relaxation times were measured using a 1.5 Tesla clinical MRI scanner. Then the corresponding relaxivities were determined.

Results: The average particle diameter of the nanocomposite was 50 to $100 \mathrm{~nm}$ based on scanning electron microscope (SEM) image. A linear relationship between relaxation rates and the $\mathrm{Fe}$ concentration of the nanocomposite was obtained. The T1 and T2 relaxivities of the nanocomposite were calculated 5.413 and $1092.1 \mathrm{mM}^{-1} \cdot \mathrm{s}^{-1}$, respectively which led to the T2/T1 relaxivity ratio of 201.75 .

Conclusion: The high T2/T1 relaxivity ratio of the iron oxide-4A nanocomposite confirms it's potential to act as a $\mathrm{T} 2$ contrast agent.

\section{Keywords}

Magnetic Resonance Imaging, Contrast Agent, Relaxivity, Iron Oxide, Nanocomposite

\section{Introduction}

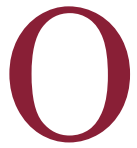

ne of the safe and non-invasive methods for in-vivo imaging and clinical diagnosis is magnetic resonance imaging (MRI) that acquires images without applying ionizing radiation. Inherent contrast in MRI depends on T1 and T2 relaxation times, and proton density of the materials or tissues [1]. In the cases that the diseased and normal tissues have similar composition, MRI inherent contrast is not sufficient to differentiate the pathologic and normal tissues. Therefore, the use of contrast agents that enhance MRI contrast is essential [2]. MRI contrast agents consist of paramagnetic or superparamagnetic metal ions which reduce the proton relaxation times and therefore produce signal intensity changes in the accumulated region [3]. This signal changes are seen on the MR images as bright regions using positive contrast agents and dark regions by applying negative contrast media [2]. The ability of a contrast agent to improve MR image contrast is determined by relaxivity which shows the changes of the relaxation rates $(1 / \mathrm{T} 1$ or $1 / \mathrm{T} 2)$ of the
${ }^{1}$ Immunology Research

Center, Tabriz University

of Medical Sciences,

Tabriz, Iran

${ }^{2}$ Department of Medical

Physics, Tabriz University

of Medical Sciences,

Tabriz, Iran

${ }^{3}$ Department of Inor-

ganic Chemistry, Faculty

of Chemistry, University

of Tabriz, C.P. 51664 ,

Tabriz, Iran

${ }^{4}$ Department of Medical

Physics, Tabriz University

of Medical Sciences,

Tabriz, Iran

${ }^{5}$ Department of Inor-

ganic Chemistry, Faculty

of Chemistry, University

of Tabriz, C.P. 51664 ,

Tabriz, Iran

${ }^{6}$ Department of MRI, Ta-

briz University of Medical

Sciences, Tabriz, Iran

${ }^{7}$ Department of Radiol-

ogy, School of Paramed-

icine, Tabriz University

of Medical Sciences,

Tabriz, Iran

*Corresponding author:

N. Gharehaghaji

Department of Radiol-

ogy, School of Paramedi-

cine, Tabriz University

of Medical Sciences,

Tabriz, Iran

E-mail: gharehaghajin@

tbzmed.ac.ir

Received: 8 April 2017

Accepted: 14 October 2017 
agent as a function of concentration.

Although traditional gadolinium chelates are usually used as paramagnetic positive contrast agents for MRI, iron oxide nanoparticles have been used as both negative and positive contrast media [4-6]. The nanoparticles advantages are including large magnetic moment, superparamagnetic behavior, metabolizable nature and high dipolar relaxivity [7]. The nanoparticles are usually coated with a biocompatible material to decrease the cytotoxicity of bare iron oxide [8], provide chemical stability and prevent aggregation of the nanoparticles from large clusters [9]. Different coating materials have been investigated for these purposes such as dextran and polyethylene glycol [10].

Zeolites are aluminosilicate crystalline materials with a regular, microporous structure containing the pores in molecular dimensions ( 0.3 to $1 \mathrm{~nm})$ and having different amounts of $\mathrm{Si} / \mathrm{Al}$ ratio [11-15]. Pore structures and crystal size are critical factors in determining the properties, for example $4 \mathrm{~A}$ zeolite is one of the synthetic zeolites with very small pore of $4 \AA$. The use of zeolite as a carrier of paramagnetic metal ions has been investigated. In a study, gadolinium zeolite has been used for gastrointestinal tract MR imaging [16]. In another study, Norek et al. reported $\mathrm{Gd}^{3+}$-loaded $\mathrm{NaY}$ zeolite potential to use as MRI contrast agent [17]. Despite iron oxide nanoparticles advantages, studies on the combination of iron oxide nanoparticles and 4A zeolite as MRI contrast agent have not been done.

The aim of this study was to evaluate the ability of iron oxide-4A (F4A) nanocomposite as MRI contrast agent. For this purpose, iron oxide nanoparticles were synthesized and encapsulated in the 4A nanozeolite pores by the hydrothermal method and magnetic resonance properties of the F4A nanocomposite were investigated.

\section{Material and Methods}

Synthesis and Characterization of
Magnetic F4A Nanocomposite

$3.12 \mathrm{~g} \mathrm{NaOH}$ was added to $45 \mathrm{ml}$ water and stirred, then $2.62 \mathrm{~g} \mathrm{Al}_{2} \mathrm{O}_{3}$ and $2.5 \mathrm{~g}$ sodium silicate were added and stirred for 7 hours. The proper amount of nano $\mathrm{Fe}_{3} \mathrm{O}_{4}(1.7 \%)$ was added to the produced gel. The mixture was carried out in an oven at $80{ }^{\circ} \mathrm{C}$ for 8 hours and washed, then dried.

$\mathrm{X}$-ray diffraction (XRD) and scanning electron microscopy (SEM) were used for the nanocomposite characterization.

\section{MTT Assay for Cell Viability}

MCF7 cells $\left(15 \times 10^{3}\right.$ cells for each well $)$ were cultured in 96-well plates with $200 \mu \mathrm{L}$ of media in each well at $37^{\circ} \mathrm{C}$. After an incubation time (24 hours), the used media were removed and the wells were washed with phosphatebuffered solution (PBS). To measure the rate of cellular proliferation, $50 \mu \mathrm{L}$ of MTT (3-(4, 5-dimetylthiazol- 2-yl)-2, 5-diphenyl- trazolium bromide) and $150 \mu \mathrm{L}$ culture medium were added to each well. The cells were incubated at $37^{\circ} \mathrm{C}$ and $5 \% \mathrm{CO}_{2}$ for 24 hours. Then the media were removed, and $200 \mu \mathrm{L}$ of dimethyl sulfoxide and $25 \mu \mathrm{L}$ Sorenson buffer were added to each well. Finally, absorbance was read using an ELISA plate reader at $570 \mathrm{~nm}$ wavelength.

\section{MRI Study}

For MRI study, suspensions of F4A with different $\mathrm{Fe}$ concentrations of $0.0084,0.0140$, 0.0219 and $0.0284 \mathrm{mM}$ were prepared by ultrasonic method. MR imaging of the samples was carried out in a 1.5 Tesla clinical MRI scanner (Magnetom Avanto, Siemens Healthcare, Germany). All glass tubes containing F4A samples were placed in the water-containing plastic container.

$\mathrm{T} 1$ relaxation time measurement was carried out using spin echo (SE) pulse sequence with repetition time (TR) values of 250, 500, 1000, 2000, and $4000 \mathrm{~ms}$ and a fixed echo time (TE) of $11 \mathrm{~ms}$. T2 relaxation times were determined using a T2-weighted multi-echo spin-echo se- 
quence with a fix TR of $3000 \mathrm{~ms}$ and four TE values of 13, 26, 39 and $52 \mathrm{~ms}$. All the images were acquired by a slice thickness of $5 \mathrm{~mm}$, a field of view (FOV) of $160 \times 160 \mathrm{~mm}^{2}$, and a matrix of $256 \times 196$ pixels.

Data analysis was carried out using DicomWorks program. Regions of interest (ROIs) were selected in each test tube image and signal intensities of the F4A samples were obtained. R1 (1/T1) and R2 (1/T2) relaxation rate values were calculated from the nonlinear fit of the mean signal intensity versus TR and $\mathrm{TE}$, respectively. The $\mathrm{T} 1$ and $\mathrm{T} 2$ relaxivities were obtained from the slope of the linear fit of the $\mathrm{R} 1$ and $\mathrm{R} 2$ relaxation rates versus $\mathrm{Fe}$ concentration of the F4A nanocomposite, respectively.

\section{Results}

Characterization of Magnetic F4A Nanocomposite

Figure 1 shows the XRD patterns of F4A nanocomposite. As shown in figure, only characteristic peaks of F4A were observed and no other structures of initial materials were present.

SEM of F4A is shown in Figure 2, there

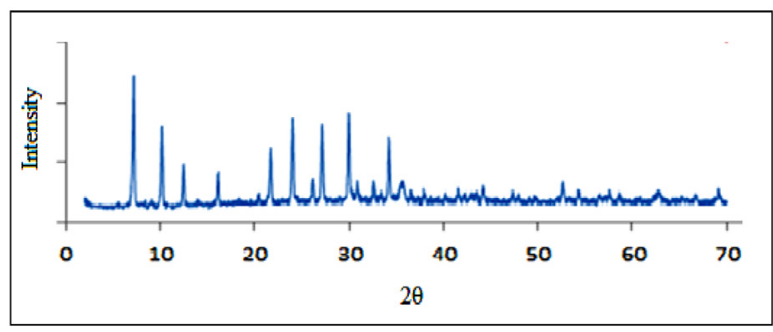

Figure 1: XRD of F4A Nanocomposite

were regular cubic shapes in crystals with different sizes (50-100 nm) of almost complete crystallinity.

\section{Cytotoxicity of F4A Nanocomposite}

Figure 3 shows the effect of increasing amounts of the F4A nanocomposite on the cells viability determined by the MTT assay

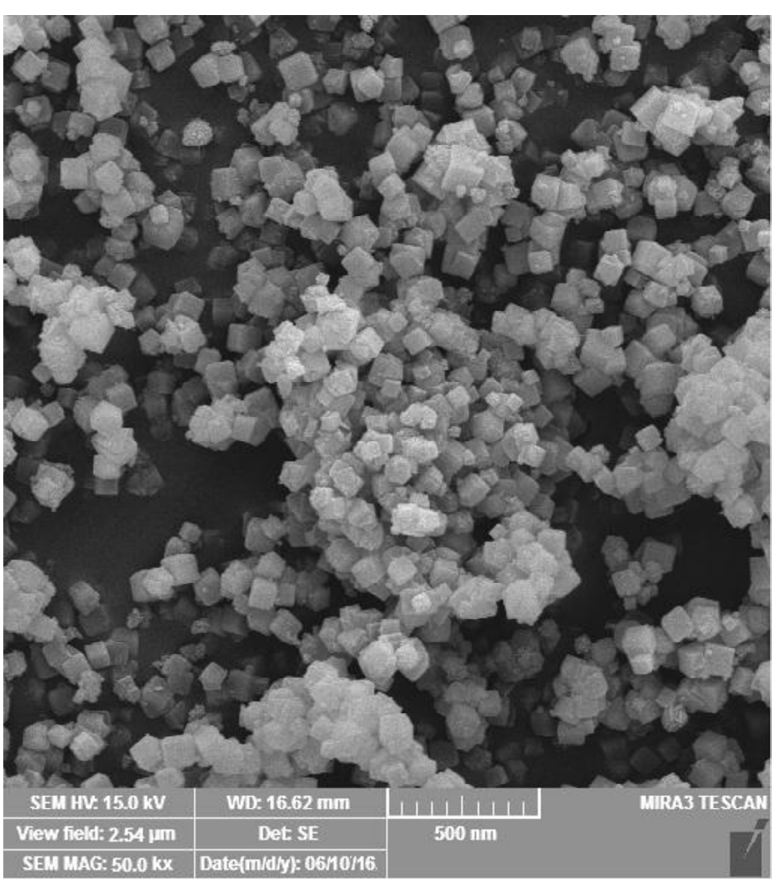

Figure 2: SEM Image of F4A Nanocomposite

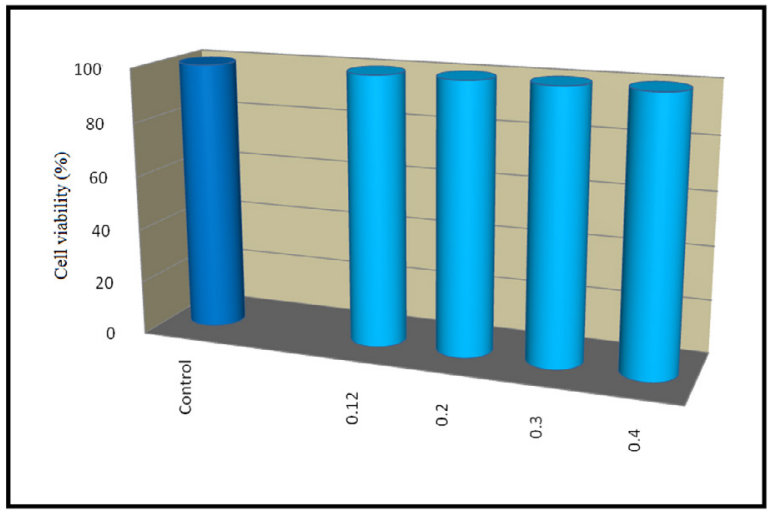

Figure 3: Representation of Cytotoxicity of F4A Nanocomposite with Different Concentrations $(\mathrm{mg} / \mathrm{ml})$

$(P>0.05)$. The differences between controls and the range of the nanocomposite concentrations were not significant which showed the F4A is not toxic to the MCF7 cell line.

\section{MRI Study}

Exponential recovery of signal with TR increasing in the all $\mathrm{T} 1$ curves, and exponential 
decay of signal with increasing of TE in the all $\mathrm{T} 2$ curves were seen. $\mathrm{T} 1$ relaxation time decreased from $3.2372 \mathrm{~s}$ for the lowest $\mathrm{Fe}$ concentration $(0.0084 \mathrm{mM})$ to $2.4894 \mathrm{~s}$ for the highest one $(0.0284 \mathrm{mM})$. The decrease for T2 was from $0.0761 \mathrm{~s}$ to $0.0285 \mathrm{~s}$ for the lowest and highest concentrations, respectively.

According to Figure 4, a linear correlation was observed between $\mathrm{R} 1$ and $\mathrm{R} 2$ relaxation rates with $\mathrm{Fe}$ concentration. The mean slopes (T1 and T2 relaxivities) were 5.413 and 1092.1 $\mathrm{mM}^{-1} \cdot \mathrm{s}^{-1}$, respectively which led to the T2/T1 relaxivity ratio of 201.75 .

\section{Discussion}

\section{Characterization of F4A Nanocom- posite}

XRD analysis results (Figure 1) indicated that the F4A nanocomposite was successfully synthesized. SEM image (Figure 2) confirmed

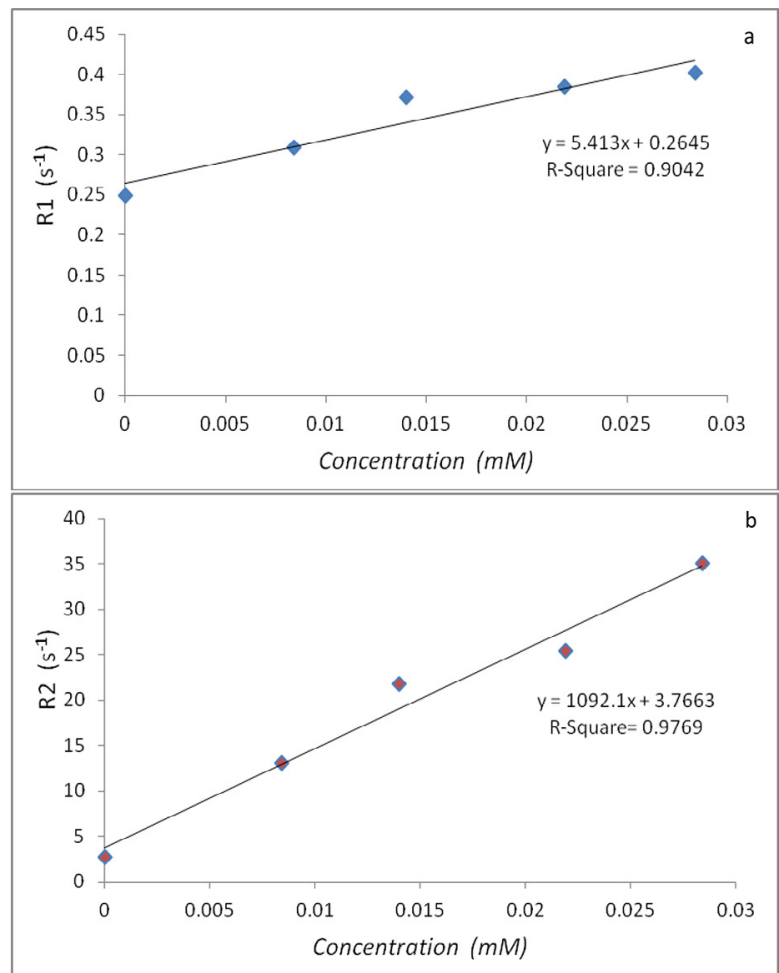

Figure 4: Linear Correlation between (a) R1 and (b) R2 Relaxation Rates with Fe Concentration of F4A Nanocomposite the nanosize and structural uniformity of the nanocomposite.

\section{MRI Study}

The results indicate that although iron oxide nanoparticles in nanocomposite structure have effects on both $\mathrm{T} 1$ and $\mathrm{T} 2$ relaxation times shortening, T2 shortening effect is dominant. Iron oxide nanoparticles produce large local magnetic fields and accelerate spin dephasing of water protons. Therefore, the high T2shortening effect results.

Similarly, as Figure 4 illustrates, T2 relaxivity of the F4A is higher than T1 relaxivity. High T2 relaxivity of the nanocomposite resulted from two main reasons; first, its structural pores and channels, and second, low ratio of $\mathrm{Si} / \mathrm{Al}$ which gives hydrophilic property to the 4A zeolite. As it seen in Figure 5, iron oxide sub-nanoclusters are capsulated in the pores and channels of the 4A zeolite and therefore they have enough access to water protons in pores and channels (positions $1 \& 2$ ) of $4 \mathrm{~A}$ zeolite. This situation enhances spin dephasing of the protons and reduces $\mathrm{T} 2$ relaxation time. Therefore, subsequently increasing of $\mathrm{T} 2$ relaxivity is resulted. It is noted that due to low concentration of iron oxide nanoparticles in nanocomposite structure, they are only placed in pores and channels, not on the surface of the zeolite (position 3).

The relaxivity ratio determines the effi-

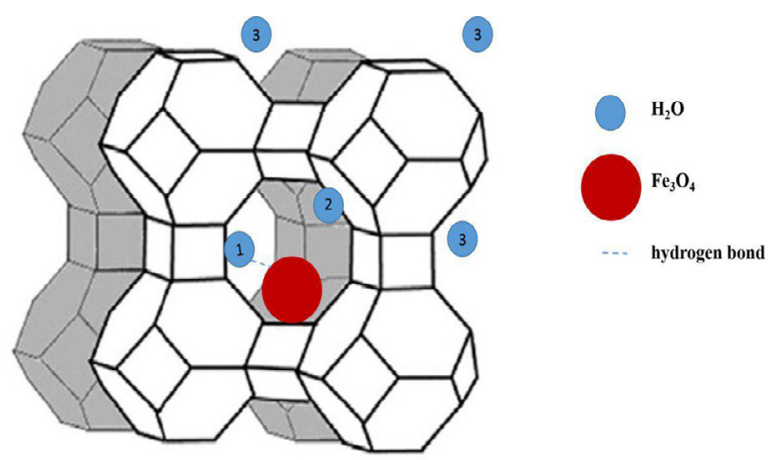

Figure 5: Position of the Water Molecules in 4A Zeolite Pores and Surface 
ciency of a contrast agent as an MRI T1 or T2 agent. If $\mathrm{T} 2 / \mathrm{T} 1$ relaxivity ratio is much bigger than 1, the contrast agent will be described as a $\mathrm{T} 2$ agent while in $\mathrm{T} 1$ contrast agents, the ratio is closed to 1 [18]. The high T2/T1 relaxivity ratio of $\mathrm{F} 4 \mathrm{~A}$ confirms that the nanocomposite can act as an MRI T2 contrast agent. The T2/T1 relaxivity ratio of F4A is significantly higher than iron oxide nanoparticles coated with organic materials in other studies. For example, the relaxivity ratio for dextran, carboxymethyl dextran (CMD), PEG-dextran [10], PEG [19] and carboxydextran [20] coated iron oxide nanoparticles were respectively reported 29.23, 11.23, 23.71, 5.7 and 9.5 in other studies which are much lower than that of F4A. Although these differences resulted from the different sizes of the iron oxide nanoparticles, and the types and thicknesses of coating materials in the studies, the $4 \mathrm{~A}$ zeolite special structure consists of pores and channels has an important role in high relaxivity ratio of the F4A due to its enough water accessibility.

\section{Conclusion}

New contrast agents designed for the optimal use of MRI are necessary. Therefore, in the current study, new zeolite based MRI contrast agent (iron oxide-4A (F4A) nanocomposite) was successfully synthesized by using the hydrothermal method. Then the ability of the F4A to use as an MRI contrast agent was investigated by the use of 1.5 Tesla clinical MRI. The results demonstrate that our nanocomposite is suitable to act as a T2 contrast agent due to its large $\mathrm{T} 2 / \mathrm{T} 1$ relaxivity ratio at clinical field strength.

\section{Acknowledgment}

Immunology Research Center of Tabriz University of Medical Sciences financially supported the current study.

\section{Conflict of Interest}

None
References

1. Joshi HM. Multifunctional metal ferrite nanoparticles for MR imaging applications. J Nanopart Res. 2013;15:1235.

2. Arsalani N, Fattahi H, Nazarpoor M. Synthesis and characterization of PVP-functionalized superparamagnetic Fe304 nanoparticles as an MRI contrast agent. Express Polym Lett. 2010;4:329-38. doi: 10.3144/expresspolymlett.2010.42.

3. Wang YX. Superparamagnetic iron oxide based MRI contrast agents: Current status of clinical application. Quant Imaging Med Surg. 2011;1:3540. doi: 10.3978/j.issn.2223-4292.2011.08.03. PubMed PMID: 23256052; PubMed Central PMCID: PMC3496483.

4. Xiao YD, Paudel R, Liu J, Ma C, Zhang ZS, Zhou SK. MRI contrast agents: Classification and application (Review). Int J Mol Med. 2016;38:131926. doi: 10.3892/ijmm.2016.2744. PubMed PMID: 27666161.

5. Corot C, Robert P, Idée JM, Port M. Recent advances in iron oxide nanocrystal technology for medical imaging. Adv Drug Deliv Rev. 2006;58:1471-504. DOI: 10.1016/j.addr.2006.09.013. PubMed PMID: 17116343.

6. Rosen JE, Chan L, Shieh DB, Gu FX. Iron oxide nanoparticles for targeted cancer imaging and diagnostics. Nanomedicine. 2012;8:275-90. doi: 10.1016/j.nano.2011.08.017. PubMed PMID: 21930108.

7. Bulte JW, Kraitchman DL. Iron oxide MR contrast agents for molecular and cellular imaging. NMR Biomed. 2004;17:484-99. doi: 10.1002/nbm.924. PubMed PMID: 15526347.

8. Shukla S, Jadaun A, Arora V, Sinha RK, Biyani N, Jain VK. In vitro toxicity assessment of chitosan oligosaccharide coated iron oxide nanoparticles. Toxicol Rep. 2015;2:27-39. doi: 10.1016/j. toxrep.2014.11.002. PubMed PMID: 28962334; PubMed Central PMCID: PMC5598369.

9. Di Marco M, Guilbert I, Port M, Robic C, Couvreur P, Dubernet C. Colloidal stability of ultrasmall superparamagnetic iron oxide (USPI0) particles with different coatings. Int J Pharm. 2007;331:197203. doi: 10.1016/j.ijpharm.2006.11.002. PubMed PMID: 17141984.

10. Oghabian MA, Gharehaghaji N, Masoudi A, Shanehsazzadeh S, Ahmadi R, Majidi RF, et al. Effect of coating materials on lymph nodes detection using magnetite nanoparticles. Advanced Science, Engineering and Medicine. 2013;5:37-45. doi: 10.1166/ asem.2013.1214.

11. Rivera A, Farıas T, Ruiz-Salvador A, De Menorval L. 
Preliminary characterization of drug support systems based on natural clinoptilolite. Microporous and Mesoporous Materials. 2003;61:249-59. doi: 10.1016/s1387-1811(03)00390-1.

12. Khatamiana M, Divband B, Daryana M. Preparation, characterization and antimicrobial property of ag+-nano Chitosan/ZSM-5: novel Hybrid Biocomposites. Nanomedicine Journal. 2016;3:268-79.

13. Bercoff PG, Bertorello HR, Saux C, Pierella LB, Botta PM, Kanazawa T, et al. Magnetic properties of Co-impregnated zeolites. Journal of Magnetism and Magnetic Materials. 2009;321:3813-20. doi: 10.1016/j.jmmm.2009.07.046.

14. Khatamian M, Divband B, Farahmand-Zahed F. Synthesis and characterization of Zinc (II)-loaded Zeolite/Graphene oxide nanocomposite as a new drug carrier. Mater Sci Eng C Mater Biol Appl. 2016;66:251-8. doi: 10.1016/j.msec.2016.04.090. PubMed PMID: 27207061.

15. Khatamian M, Divband B, Jodaei A. Degradation of 4-nitrophenol (4-NP) using ZnO nanoparticles supported on zeolites and modeling of experimental results by artificial neural networks. Mater Chem Phys. 2012;134:31-7. doi: 10.1016/j.matchemphys.2012.01.091.

16. Young SW, Qing F, Rubin D, Balkus KJ, Jr., Engel
JS, Lang J, et al. Gadolinium zeolite as an oral contrast agent for magnetic resonance imaging. $J$ Magn Reson Imaging. 1995;5:499-508. PubMed PMID: 8574032.

17. Norek M, Neves IC, Peters JA. $1 \mathrm{H}$ relaxivity of water in aqueous suspensions of $\mathrm{Gd} 3+-$ loaded $\mathrm{NaY}$ nanozeolites and AITUD-1 mesoporous material: the influence of Si/Al ratio and pore size. Inorg Chem. 2007;46:6190-6. doi: 10.1021/ic700699n.

18. Liu CL, Peng YK, Chou SW, Tseng WH, Tseng YJ, Chen $\mathrm{HC}$, et al. One-step, room-temperature synthesis of glutathione-capped iron-oxide nanoparticles and their application in in vivo T1-weighted magnetic resonance imaging. Small. 2014;10:3962-9. doi: 10.1002/smll.201303868.

19. Dai L, Liu Y, Wang Z, Guo F, Shi D, Zhang B. Onepot facile synthesis of PEGylated superparamagnetic iron oxide nanoparticles for MRI contrast enhancement. Mater Sci Eng C Mater Biol Appl. 2014;41:161-7. doi: 10.1016/j.msec.2014.04.041. PubMed PMID: 24907749.

20. Pereira C, Pereira AM, Rocha M, Freire C, Geraldes CF. Architectured design of superparamagnetic Fe 304 nanoparticles for application as MRI contrast agents: mastering size and magnetism for enhanced relaxivity. Journal of Materials Chemistry B. 2015;3:6261-73. doi: 10.1039/c5tb00789e. 